\title{
Advanced selective key point detection techniques
}

\author{
Original \\ Ahmed S. Amein ${ }^{1}$ and Abdel Hameed El-Tanany ${ }^{2}$ \\ Article \\ ${ }^{1}$ Radar Department, Electronic and Electrical Engineering, Military Technical College, \\ ${ }^{2}$ Space Centre, Intelligence Sector, Egyptian Ministry of Defence, Cairo, Egypt
}

\section{Keywords:}

Corner detector, feature, Harris reflector, image registration, key point.

\section{Corresponding Author:}

Ahmed S. Amein, Radar Department, Electronic and Electrical Engineering, Military Technical College, , Cairo, Egypt, Tel: +201113041333

Email: asamein@mtc.edu.eg.

\begin{abstract}
SAR imaging needs precise registration process to get recompensation or setoff between two or more images for a certain area. Generally, operational goal of registration process is a geometrical transformation for the input leading to geometrically agreement for input images. Coregistration step can be used in a lot of fields as change detection, mosaicking, super-resolution images creation, ... etc. Registration process can be classified into two main classes: Classical or Automatic methods. For the classical approach, the anchor points are picked out manually and then the transformation modeling is performed, that leads to more time consuming and low output accuracy. Automatic detection of these anchor points aims to recover the functionality of non-automated processes. Registration process should recover many problems such as illumination changes, variations of intensity, Different sensors, noise, ... etc. Its applications are mainly dependent on errors, such as multi temporal; multi view; or multi modal, occurred during capturing process. Feature detection, as a step of image registration process, obtains a group of anchors, that are stable and informativeness, or patches under varying conditions. Also, it is critical for the detector to have robustness against viewpoint changes, brightness changes, and other deformations. Detailed discussion and investigation are paid, in this manuscript, for the common corner detectors helping to be familiar with the various applied techniques for feature detection. Our goal is to examine a combination hypothesis of these methods into an advanced selective one.
\end{abstract}

\section{INTRODUCTION}

Detection process of features is very important to represent the image ${ }^{[1-9]}$. There are two main approaches for automatic registration. Firstly, Area Based Matching methods (ABM), may be called as coarse co-registration, is pixel-to-pixel registration process. It includes methods as Fourier, correlation, mutual information, and optimization. ABM methods emphasize the existence of features rather than their detection, i.e. no features are detected. Secondly, Feature Based Matching methods (FBM) or fine co-registration as may be called, is subpixel-tosubpixel process. Each one of these two approaches have its own advantages and drawbacks ${ }^{[10,11,12]}$. Features may be points (corners, interest), edges, blobs, texture, or color $^{[1-5,8,9,11,12]}$. In general, there are two types of features that can be detected, global and local features. Generally, the content of image is depicted totally using global features, whereas the process of image keypoints detection requires the local one's usages, their representation is as shown in figure $1^{[11,12,13,14,15]}$.

Global features have the drawback of image clutter and occlusions which limit the usefulness of the description. To handle the limitations of global features, its desired to do one of the following: 1) image segmentation ${ }^{[16,17]}$ only for a few regions or 2) image sampling ${ }^{[18,19]}$ for different image subparts and this bordered the extent of potential applications ${ }^{[11,13]}$. The expectation of local features being more useful than global ones is based on their specifications which are mainly dependent on ascendant performance, structure stability, and informativeness property ${ }^{[8,9,20,21]}$. To get correspondences among images, it is required to distinguish a set of noteworthy points in each one ${ }^{[22]}$. Various techniques of feature detection have been proposed and were planned to adequate a certain 
purpose as staff matching ${ }^{[1,2,11]}$. The best execution is mainly dependent on the parameters of the detector ${ }^{[11-14,}$ 23]. The detectors must have specific characteristics depending on the application as robustness, repeatability, accuracy, generality, efficiency, and quantity. Good feature should be repeatable, informativeness, localized ${ }^{[2,}$ 11-16]. It must be noticed that not all the properties or characteristics of the detector can be fulfilled simultaneously for example properties of distinctiveness and locality ${ }^{[14,15,24]}$.

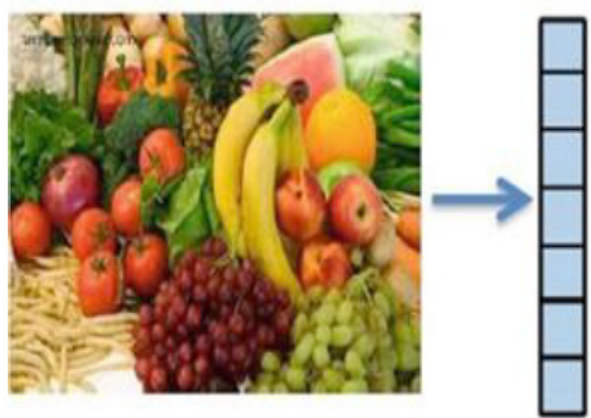

(a) Global features

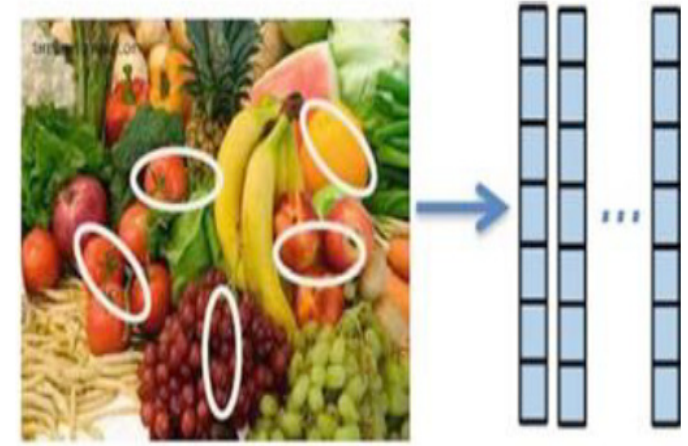

(b) Local features

Fig. 1: Representation of image features

Corner can be illustrated as the intersection point of two or more different directions of rims ${ }^{[11,13,24]}$. Accuracy of features detection, specially corners, is difficult under imperfections of data such as radiometric (illumination changes and/or different sensor characteristics) and geometric (scaling, translation, rotation, affine, projective, or nonlinear differences) differences. So, it is desired to detect the same features in the images in spite of these imperfections. Scaling, translation, and rotation problems can be easily recovered since there is no change in the shape of information. But; with respect to affine, projective, and nonlinear problems, the process is more difficult to be handled since the shape of information changed $^{[12,13]}$. Therefore, features detection methodology should be insensitive to common geometric and radiometric changes ${ }^{[1,2,4,10-16,24]}$.

Commonly used techniques to acquire corners are contour curvature-based methods, intensities based on derivatives methods, exploitation of color information, human visual system, model-based methods, segmentation-based methods, and machine learning techniques $^{[1,2,4,11,14-16]}$. Examination of the performance of a corner detector, basically; started with artificially created images with various types of junctions associated with different lengths, contrasts, angles etc. This approach can make simplification for the evaluation process but cannot model all imperfections which affect the performance of a detector in a real case, so; the results of performance are often over-optimistic ${ }^{[1,14,15]}$. Evaluations of both performance and quality of different key point detection methods were discussed ${ }^{[25-26]}$. Finally; Merging several key point detection techniques is useful if it is handled well[ ${ }^{[24,27]}$.

\section{COMMON CORNER DETECTORS}

Detection operation of feature can be classified into three classes of corner detection template, contour, and direct detection ${ }^{[24,28,29]}$. Also, it can be categorized according to the operating scale into single scale, multiscale, and affine invariant ${ }^{[1,11,12,24]}$. As mentioned before, the aim of this paper is helping readers who is looking forward to be familiar with the different applied techniques used for feature detection.

\section{II.1. Moravec Corner Detection}

Moravec's detector ${ }^{[30]}$ is a single-scale Detector. It is one of the earliest approaches that specifies a key point to be that of low essence similarity. Each image's pixel is investigated by achievement of correlation among overlapping patches such that, the omni-directional correlation's strength disclosures data about this pixe $^{[11-14,24]}$. Strength of corner is acquainted, as the smallest sum of squared differences (SSD), between the patch and its neighbors for main directions (horizontal, vertical, and diagonals), where the moment at which SSD reaches a local maximum; a corner (interest point) is detected ${ }^{[31]}$. Figure 2 depicts how to achieve the nonmaximum suppression operation ${ }^{[11]}$. 


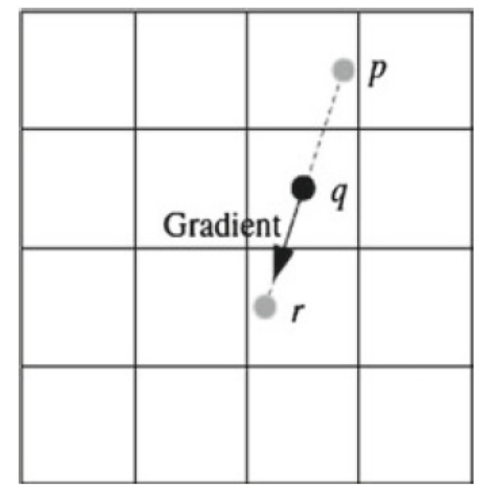

Fig. 2: Non-maximum suppression operation

Both existence of noise, can be recovered by smoothing, and increasing the size of window reduce the number of detected feature points and subsequently number of symmetric points. When calculating differences in eight directions, the output befits invariant to rotations that are a multiple of 45 degree $^{[12]}$.

Advantages of Moravec's detector are as following: a) detecting the lump of the corners, and b) requires less computation than finding the central moment at a window of the same size. While, the disadvantages of Moravec's detector are: a) it is not isotopic, and

b) its computing is shrill[ ${ }^{[11-14]}$.

\section{II.2. Harris Corner Detector}

Harris corner detector ${ }^{[32]}$ is a single scale derivativesbased detector that had been advanced to handle the of Moravec's constraints by getting the correlation changes for different orientations. It can be considered as a combination of both edge and corner detectors, mainly for gray scale image ${ }^{[11-14,24,33]}$. The gist idea is calculation of the eigenvalues for a slight patch. Then, using the two highest eigenvalues to estimate a specific function which is used together with a threshold to detect the corners which have an ill-defined gradient as shown in figure $3^{[15]}$. Its main target, for feature orientation, is to obtain the fastest and slowest change directions by using local directional derivatives covariance matrix ${ }^{[23,33]}$. Also, improvements over the Moravec method based on Harris were done by Schmid $^{[34]}$ and B. Sirisha ${ }^{[35]}$ developed a detector to be utilized for the color information ${ }^{[11,33]}$.

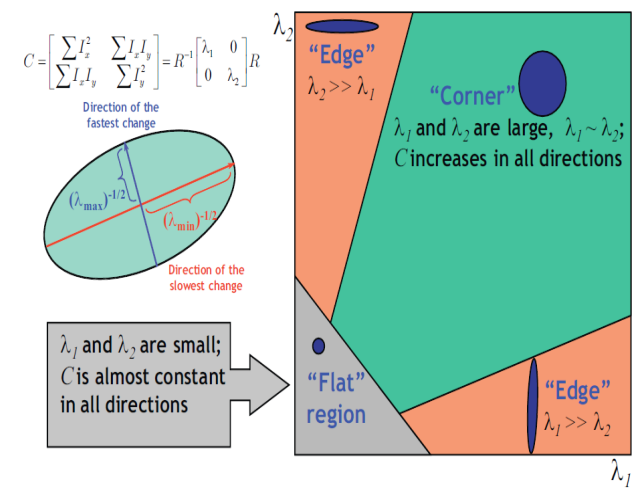

Fig. 3: Illustration of gist idea of Harris detector.
Harris corner detector has many advantages as following: 1) it has robustness against variations of rotation, translation and illumination ; 2) it is high discriminative, localized, and repetitive ; 3) it is the most stable one in many independent evaluations ; 4) it provides high rotational invariance repeatability ${ }^{[11-14,23,24,36,37]}$. The Disadvantages of Harris corner detectors are: 1) it may be sensitive to noise ;2) it has more expensive computations requirements ; 3 ) it is sensitive to high scale changes ${ }^{[13,14,23]}$.

Shi, Tomasi ${ }^{[38]}$ was developed as an optimization based on Harris method. Shi, Tomasi allow the usage of the minimum eigenvalues for differentiation and considerably thus regulates and facilitates the computation of Harris $^{[11,23]}$.

Since developing the robustness of the detector to different types of transformations requires being invariant to affine transformations, so; Harris Laplace / Harris Affine ${ }^{[39,40]}$ were developed, based on Harris method, to act as a multi-scale affine invariant corner detector. The main idea based on a blend of Harris method and a gaussian scale space representation allowing detection of features over a range of scales ${ }^{[1-14,23]}$.

Their advantages are: 1) it provides a high informative group of anchors in the scale dimension ; 2) it has a robustness against changes of scale ; 3) Also, it has a robustness against noise, illumination ; 4) it has high repeatability rate. Their disadvantages are: 1) reduction the excessive number of anchor points comparing to other multi-scale techniques as Laplacian of Gaussian (LoG) or Difference of Gaussian (DoG), and 2) it has bad performance with respect to affine transformations ${ }^{[11,12,23]}$.

Tuytelaars and Van Gool ${ }^{[41,42]}$ developed, based on Harris method, a different approach depending on local invariants which distinguish neighborhoods of interest points. The cause backwards this tendency is that rims are counted as settled features that can be distinguished over multiple changes as illumination, viewpoints, scaling. Further, by utilizing the geometry of the edge, the dimensionality of the issue can be safely diminutived ${ }^{[11-14,23]}$.

\section{II.3. SUSAN Corner Detectors}

Susan corner detector ${ }^{[43]}$ is a single scale detector which does not smooth the image or use locative derivatives. It uses a morphological approach as an unlike way rather than estimating gradients of local which may be sensitive to noise and more cost computations. Smallest Univalue Segment Assimilating Nucleus (SUSAN) has been used as a corner detection and edge detection besides noise suppression where an annular disguise is utilized concerning each pixel, and checking the gray accounts concerning all pixels inside the disguise then comparing with that of central one ${ }^{[11-15,23]}$. Corners can be distinguished as the locations where the number of same intensity records in a regional vicinity catches a local floor and beneath a specified onset as shown in figure 4 where $\mathrm{A}$ is an edge, $\mathrm{B}$ is a corner, and $\mathrm{C}$ is neither an edge nor a corner ${ }^{[13,23]}$. Increasing 
robustness can be achieved using weighting coefficient where Univalue Segment Assimilating Nucleus (USAN) represents a grouping of the vicinity, having similar value as the central one, together ${ }^{[11-15,23,33]}$. It has a high performance in spite of noise existence since it does not call derivative ${ }^{[23,44]}$.

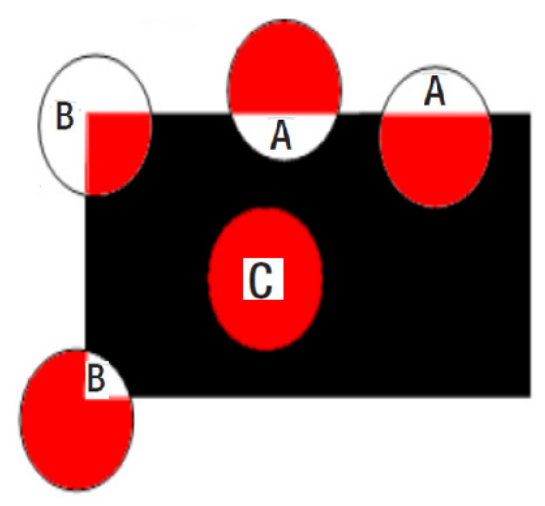

Fig. 4: Interest point Computations of SUSAN method

The advantages of SUSAN corner detector are as following: 1) its computations are fast since no derivatives are used, 2) it can be used as corner and edge detector in addition as image noise reduction, 3) it has good repeatability rate, 4) it is more efficient. 5) It has robustness against changes of rotation, translation and illumination. The disadvantages are: 1) it is sensitive to scaling and affine, 2) specifying a steady onset is not good for generic applications; 3 ) it has low noise sensitivity, low robustness, and low localization, 4) it is less discriminative $\mathrm{e}^{[11-15,23,45]}$.

\section{II.4. Förstner Corner Detector}

Förstner corner detector ${ }^{[46]}$ is a single scale detector, developed in a manner to register the corner location up to sub-pixel precision. The main goal is finding the best solution for the point highest to whole corner tangential paths, as shown in figure 5 , in a predefined overture. For the ideal corner, all the tangential lines cut across at a unique point and this is its main gist. Förstner corner detector had been automatically adapted by Lindeberg ${ }^{[47,48]}$ to have the ability to be multiple scale for counting the gradients of image in spite of noise existence ${ }^{[11,13]}$.

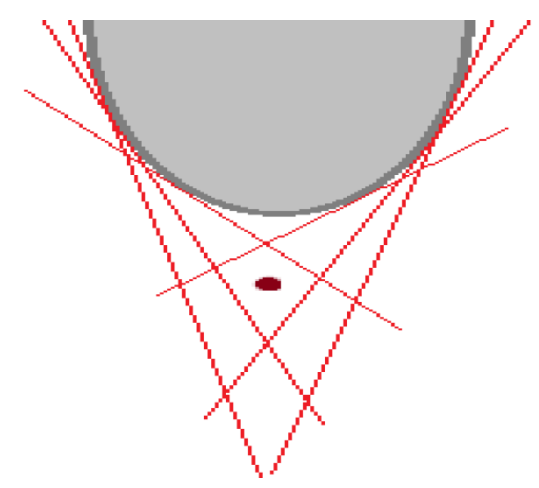

Fig. 5: Förstner corner detector

\section{II.5. Robust Fuzzy Rule Corner Detector}

Corner detection technique for general purpose, with high performance and efficiency, is a challenge. Starting from this point, a few fuzzy methods have particularly discussed this problem ${ }^{[11,13,14,23]}$. An algorithm has been proposed by Banerjee and $\mathrm{Kundu}^{[49]}$ to extract significant corner points in gray level where the cornerness measuring for each candidate is counted by ways of the vague rim strength. Changing threshold value causing various groups of corners (fuzzy) as shown in figure 6. The main advantage of Fuzzy Rule corner detector is that it may be easily used for detecting different types of anchors. Using different types of detectors, at various stages, causes a high load of computations. This can be considered its main drawback $^{[13,14,25]}$.

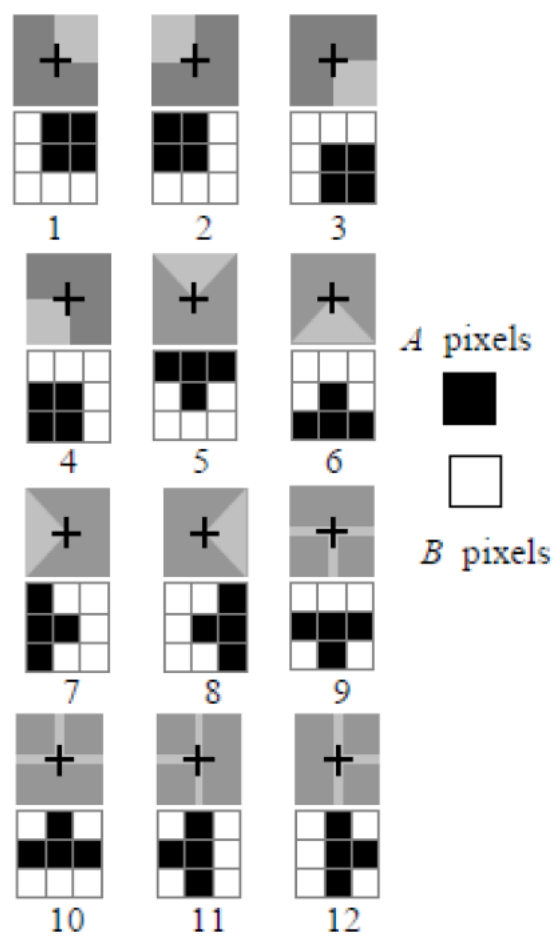

Fig. 6: Different Fuzzy Corner set

\section{II.6. Hessian Detector}

Hessian detector ${ }^{[50,51]}$ is a single scale blob detector, also it can be called Determinant of Hessian (DoH) which investigates for positions that offer powerful derivatives in two rectangular directions. Its main idea stands behind searching for a point, center of searching window, whose determinant of hessian, matrix containing 2 nd derivative, is maximum as shown in figure 7 . Then, non-maximum suppression is applied retaining only anchors whose rating is greater than that of all eight-spot nearness inside the specified overture. Finally, all lasting points, whose value is above a specific predefined threshold, are returned by the detector ${ }^{[11,14,15,23,24]}$. DoH method is applicable for the generic SURF manner ${ }^{[52]}$. The advantages of hessian detector are:1) regions with strong texture variation could 
be returned with many responses, 2) it can provide further interest points that result in a densely objects, 3 ) it has good performance and repeatability. The drawbacks of hessian detector are: 1) it is sensible to changes of scale, 2) it is sensitive to noise leading to low localization accuracy causing the local maxima to be less stable ${ }^{[11,13,14,23,40]}$.

Hessian-Laplace detector ${ }^{[40,53]}$ was developed to act as a changeless scale blob detector which is a blend of hessian detector and a gaussian scale space illustration. The core is that it operates on local extrema at various scales using DoH for spatially localized, and the Laplacian for localization of the scale. Mikolajczyk, K. ${ }^{[54]}$ is designed to be insensible to affine, it utilizes Harris based detector, integrating key points from different scales in a pyramid with some reiterated election gauge, and matrix of
Hessian [11, 12, 23]. Mikolajczyk and Schmid ${ }^{[53]}$ concluded that the response of hessian detector exists if significant variations over any two perpendicular ways occurred. Also, for a single image, the repeatability, discernment more reliable regions, of hessian affine approach better than that of Harris affine one. In addition, it complies good to regions which have many corners, for example, buildings scenes ${ }^{[11-15,23,24]}$.

The advantages of hessian affine are 1) it has the ability of extraction large number of features, 2) using spatial and scale localization is more suitable for scale estimation, and 3) It has a high stability compared to the Harris one. Although these advantages, hessian affine has a drawback that its repeatability is low compared to Harris-Laplace ${ }^{[11,52]}$.

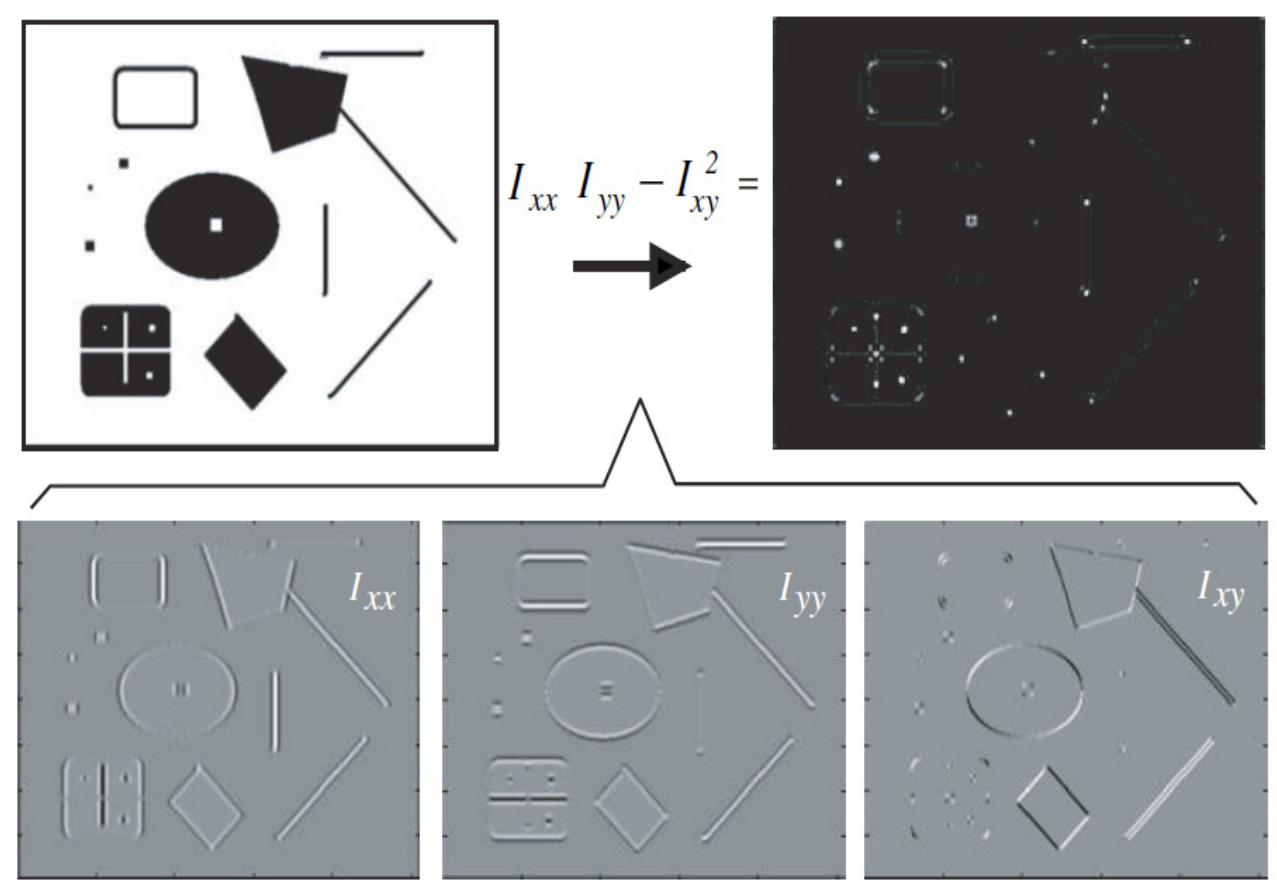

Fig. 7: Components of both Hessian matrix and determinant

\section{II.7. FAST Detector}

Rosten, E., Drummond ${ }^{[55]}$ proposed Features from Accelerated Segment Test (FAST) detector which is a uniscale keypoint detection. Interest points are categorized by utilizing a part check to the pixel intensity. This can be achieved by concerning a ring (bresenham circle) consisting of sixteen pixels, as base of computation, around the interest point ${ }^{[11,14,23,24]}$.

The key point can be detected if its intensity rate (I) is larger or junior than the value of a collection of (n) neighboring pixels in bresenham circle as shown in figure 8 , taking into account a predefined threshold ${ }^{[11]}$. Rosten, E., Drummond ${ }^{[56]}$ developed a test, whose speed is high, to eliminate a considerable number of points which are not corner by investigating only the pixels numbers 1, 5, 9 and 13. If three of these four test pixels are luminous or darker than the intensity value, then a corner can only exist as a first step. For final result, the remaining pixels are examined. FAST detector is similar to SUSAN, but uses a smaller window size in addition to the fact that only some of pixels are examined instead of all of them. It can be rated as a proportion of the local binary pattern $\operatorname{LBP}^{[14,23]}$.

Fast detector has many advantages as it has high performance, high speed, efficiently computations and faster matching, a good precise, high repeatability, and low averaging out noise. Also, it is fast compared to the other ones. It suffers from being sensitive to high rates of noise depending on an onset, probability to respond at specific oratories, and also it is sensible to scale changes ${ }^{[1,56]}$. 


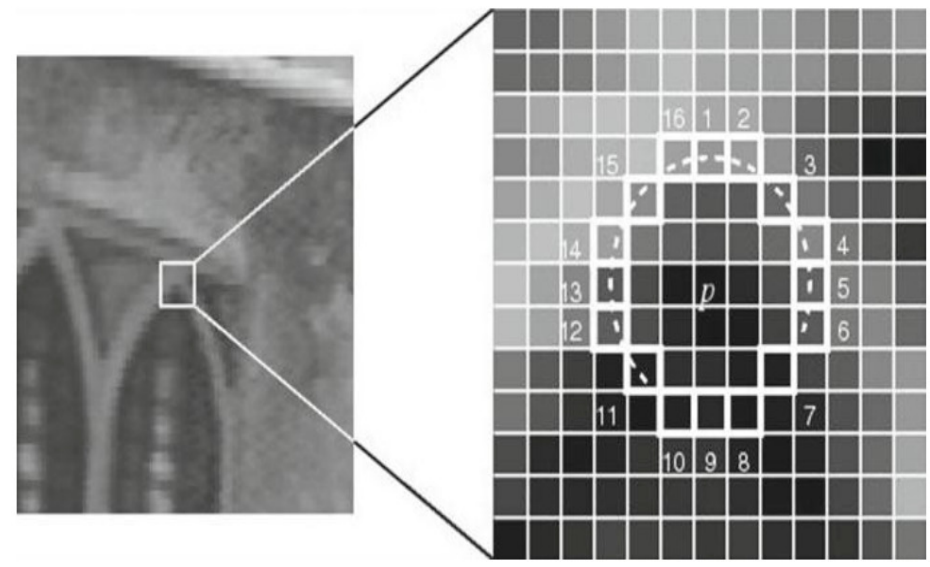

Fig. 8: Illustration of operation of FAST corner detector

\section{II.8. Laplacian / LoG / DoG Detectors}

Laplacian operation, is a manner for computing the extreme average of change (derivative) in a pixel. It is described for a gray-scale image ${ }^{[11,14,23,24]}$.

Laplacian of Gaussian (LoG $)^{[57]}$ is a detector dedicated for various scales which is simply combines Laplacian operation over an area that had been treated utilizing a smoothing Gaussian kernel to converge the rim as shown in figure $9^{[12]}$. This means that LoG is a linear series of 2nd derivatives and a blob detector. The operator's output depends strongly on the link between size of the structures of blob and the size of smoothing Gaussian kernel where the scale is controlled by the Gaussian standard deviation by varying the blurring amount ${ }^{[11,23]}$. LoG at a pixel in the image becomes locally maximum or minimum when the pixel represents the center of a dark or bright spot respectively ${ }^{[12,14]}$.

Lindeberg $^{[48]}$ developed an automatically method, considered to be a local curvature detection, for capturing blobs of different size where the automatic selection of the scale and a multi-scale method is developed such that both curvature of local surface and the magnitude of the gradient are high. It is important to mention that the local curvature methods, as Rosenfeld and Bretzner ${ }^{[58,59]}$, were firstly known to be accurate and reliable for focusing anchor points over the changes of the scale ${ }^{[11-14,23,24]}$.

Advantage of LoG are: 1) it is invariant to rotation, 2) it provides somewhat a good rating for other features such as corners, ridges, etc., 3) the detected features can represent extended and splitting structures. Disadvantage of LoG are: 1) its computation is time consuming, 2) less stable, 3) more sensitive to noise $\mathrm{e}^{[11,12]}$.

Difference of Gaussian (DoG) ${ }^{[60]}$ can be considered as a multi-scale detector which is utilized to speed up computation of LoG, i.e. DoG provides a nearest access to LoG as shown in figure 10 . This can be accomplished using subtraction of the neighbouring levels of the scale of the Gaussian pyramid breaked up by a certain operator without needing for convolution. The operation of DoG efficiently detects the nonvarying keypoints from scale-space, while it suffers from the same drawbacks of LoG ${ }^{[11,2340]}$.

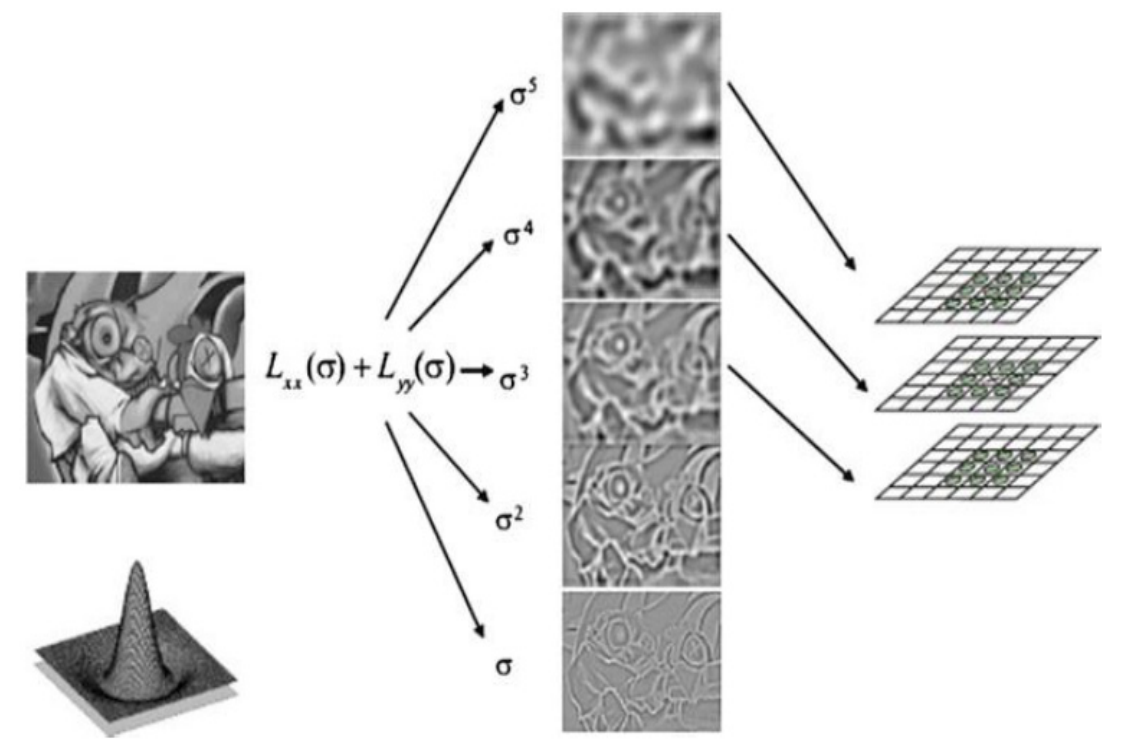

Fig. 9: Laplacian of Gaussian (LoG) operation 


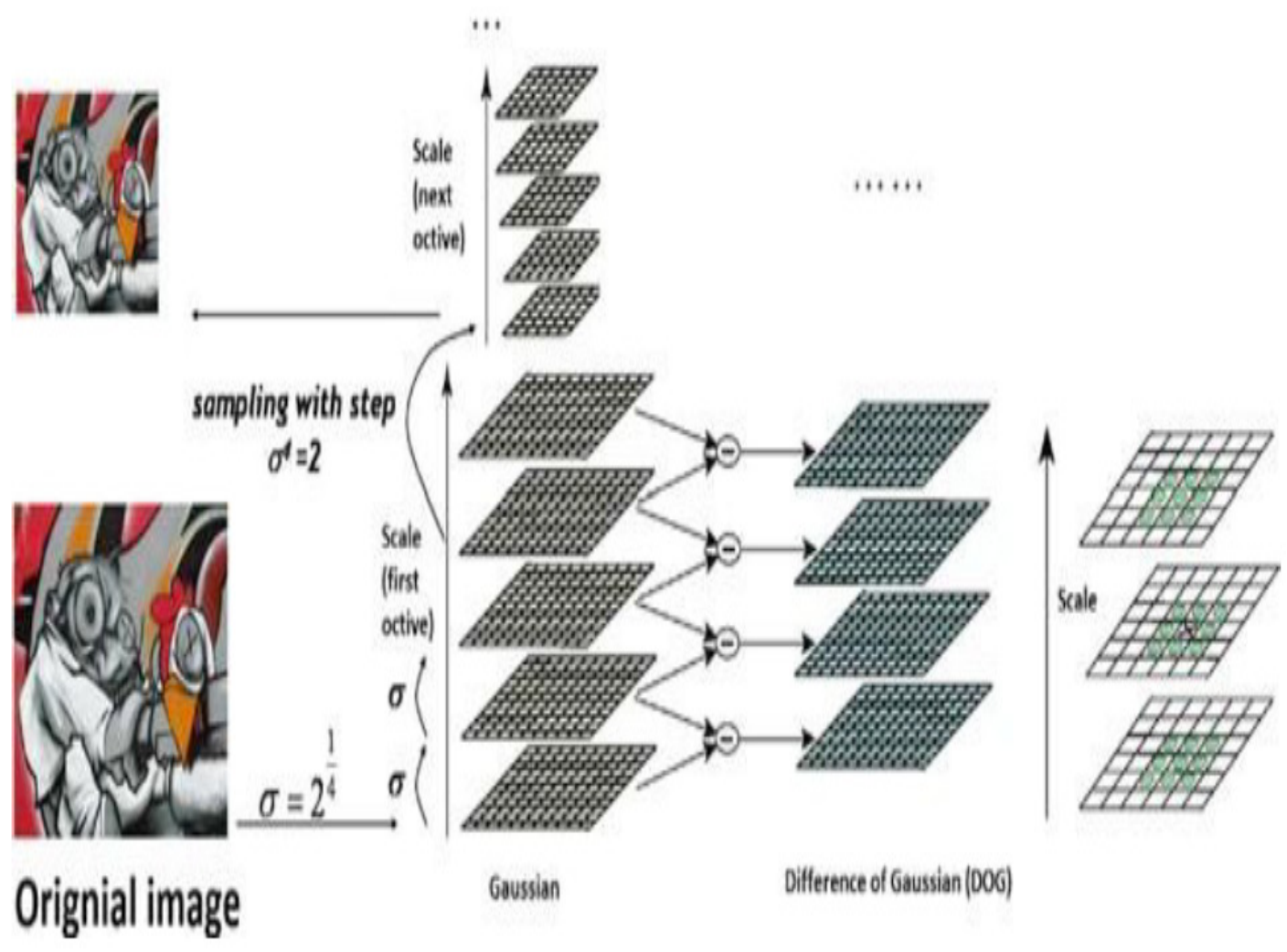

Fig. 10: Difference of Gaussian (DoG) operation

\section{II.9. Salient Regions (Entropy) Detection}

Kadir and Brady ${ }^{[61,62]}$ developed a novel methodology which is established on thought that key points over a multi-scale should present a local characteristics (entropy) that are unexpected compared to the neighborhood region. The entropy method is mainly based on locally maximizing the information content, therefor, the most distinctiveness points can be identified ${ }^{[11,12,24,23]}$. The most important note for the entropy method is being a rotationally invariant feature since location of pixel are not used in the calculation, therefore the entropy window will not alternate by rearrangement its pixels with respect to the centered window ${ }^{[62,24]}$

\section{II.10. Gabor-Wavelet Detector}

Gabor-Wavelet detector ${ }^{[29,63]}$ can be considered as key point detector operating in multi-scale relying on the basis of Gabor wavelet which is stimulated convolution kernels in the modality of plane waves bounded by a Gaussian envelope function ${ }^{[11,12,29,63]}$. Gabor-Wavelet has many advantages as: 1) it provides the highest and the best ruling for spatial and space frequency domains, 2) It has the ability for improving features of low level like peaks, ridges, etc., 3) It has the capability of extraction of points from different scales, and 4) Its performance is categorized with precision and modification to multiple geometric transformations ${ }^{[11,12,29,63]}$.

\section{II.11. Morphological Interest Regions}

Morphological operations ${ }^{[64-68]}$ can be non-varying with respect to both rotation and scale. The prime usage of mentioned method based on creating an interest region on gray scale, binary, or color images. It is important to notice that the process of preparation both gray scale and color images requires many forms of pre-processing operations ${ }^{[23,62]}$. The binary thresholding necessitates a part of work to correctly regulate the arguments for a specific exercise to get the highest performance. Generally, the morphological operations alone are not sufficient ${ }^{[1,23,62]}$. Since Maximally Stable Extremal Regions (MSER) ${ }^{[69]}$, feature descriptor technique, is based on the principle of gathering the groups of pixels at maximum or minimum locations, so the morphological interest regions method is similar to MSER, but it is important to know that MSER does not utilize the morphology operators ${ }^{[23]}$.

\section{CONCLUSION}

Feature detection, as a step of image registration process, obtains a group of anchors, that are stable and informativeness, or patches under varying conditions. It is critical for the conventional detector to have robustness against viewpoint changes, brightness changes, and other deformations. Already existed FBM algorithms suffers from different limitations or $\mathrm{c}$ deficiencies in dealing with 
certain SAR image sets. Our combined, fully automated, advanced selective feature based key method helps to overcome these limitations and deficiencies. A complete discussion of this selective technique had been addressed.

\section{REFERENCES}

[1] Mahmoud Hassaballah, Khalid M. Hosny, "Recent Advances in Computer Vision Theories and Applications", Studies in Computational Intelligence, Volume 804, ISBN 978-3-030-02999-9, (c) Springer Nature Switzerland AG, 2019.

[2] Ertu grul BAYRAKTAR , P nar BOYRAZ, "Analysis of feature detector and descriptor combinations with a localization experiment for various performance metrics", Turkish Journal of Electrical Engineering and Computer Sciences (Turk J Elec Eng Comp Sci), V25, pp: 2444-2454, 2017.

[3] C. P. Dalmiy, V. S. Dharun, "A Survey of Registration Techniques in Remote Sensing Images", Indian Journal of Science and Technology, Vol 8(26), ISSN (Print): 0974-6846, October 2015.

[4] Aboul Ella Hassanien, Mohamed F. Tolba, Khaled Shaalan, Ahmad Taher Azar "Advances in Intelligent Systems and Computing", Proceedings of the International Conference on Advanced Intelligen Systems and Informatics, Volume 845, ISBN 978-3-319-99009-5, C Springer Nature Switzerland, 2019.

[5] Dibyendu Mukherjee, Q. M. Jonathan Wu, Guanghui Wang, "A comparative experimental study of image feature detectors and descriptors", Machine Vision and Applications, V26, p: 443-466, DOI 10.1007/s00138-015-0679-9, (C) Springer-Verlag Berlin Heidelberg, 2015. [6] Reshmi Krishnan, Anil. A. R, "A Survey on Image Matching Methods", International Journal of Latest Research in Engineering and Technology (IJLRET), Volume 2 Issue 1, ISSN: 2454-5031, PP 58-61, January 2016.

[7] Niangang Jiao, Wenchao Kang, Yuming Xianga, Hongjian You, "A NOVEL AND FAST CORNER DETECTION METHOD FOR SAR IMAGERY", The International Archives of the Photogrammetry, Remote Sensing and Spatial Information Sciences, Volume XLII-2/W7, Wuhan, China, September 2017.

[8] A.A. Karim, E. F. Nasser, "Improvement of Corner Detection Algorithms (Harris, FAST and SUSAN) Based on Reduction of Features Space and Complexity Time", Engineering and Technology Journal Vol. 35, Part B. No. 2, 2017.

[9] Ehab Salahat, Murad Qasaimeh, "Recent Advances in Features Extraction and Description Algorithms: A Comprehensive Survey", arXiv:1703.06376v1 [cs.CV] 19 Mar 2017.

[10] Chen, J., Shan, S., He, C., Zhao, G., Pietikäinen, M., Chen, X., Gao, W., "WLD: a robust local image descriptor", IEEE Transactions on Pattern Analysis and Machine Intelligence, V 32 , Issue 9 , pp: 1705-1720, September 2010.

[11] M. Hassaballah and Ali Ismail Awad, "Image Feature Detectors and Descriptors: Foundations and Applications", Studies in Computational Intelligence 630, ISBN 978-3-319-28852-9, (C) Springer International Publishing Switzerland, 2016

[12] Arthur Ardeshir Goshtasby, "Theory and Applications of Image Registration", First Edition, JohnWiley and Sons Inc., ISBN 9781119171720, JohnWiley and Sons, Inc., 2017.

[13] Trupti P. Patel, Sandip R. Panchal, "Corner Detection Techniques: An Introductory Survey", International Journal of Engineering Development and Research (IJEDR), Volume 2, Issue 4, ISSN: 2321-9939, 2014.

[14] Tinne Tuytelaars, Krystian Mikolajczyk, "Local Invariant Feature Detector: A Survey", Foundations and trends in Computer Graphics and Vision, Volume 3.3, ISBN 978-1-60198-138-7, the essence of Knowledge, 2008.

[15] Kristen Grauman and Bastian Leibe, "Visual Object Recognition SYNTHESIS LECTURES ON COMPUTER VISION \# 1", Visual Recognition, 2010.

[16] C. Carson, S. Belongie, S. Greenspan, and J. Malik, "Blobworld: Image segmentation using expectation-maximization and its application to image querying," IEEE Transactions on Pattern Analysis and Machine Intelligence, vol. 24 , no. 8, pp. 1026-1038, 2002

[17] F. Schaffalitzky and A. Zisserman, "Viewpoint invariant texture matching and wide baseline stereo", in Proceedings of the International Conference on Computer Vision, pp. 636-643, 2001.

[18] P. Carbonetto, N. de Freitas, and K. Barnard, "A statistical model for general contextual object recognition," in Proceedings of the European
Conference on Computer Vision ECCV, part I, pp. 350-362, 2004.

[19] J. Winn, A. Criminisi, and T. Minka, "Object categorization by learned universal visual dictionary," in Proceedings of the International Conference on Computer Vision, vol. 2, pp. 1800-1807, 2005.

[20] Jégou, H., Perronnin, F., Douze, M., Sánchez, J., Pérez, P., Schmid, "C.: Aggregating local descriptors into a compact code", IEEE Trans. Pattern Anal. Mach. Intell. 34(9), 1704-1716, 2012.

[21] Bianco, S., Mazzini, D., Pau, D., Schettini, R., "Local detectors and compact descriptors for visual search: a quantitative comparison", Digital Signal Process. 44, 1-13, 2015

[22] Bouchiha, R., Besbes, K., "Comparison of local descriptors for automatic remote sensing image registration", Signal, image and video processing (SIViP), (C) Springer-Verlag London, SIViP 9(2), 463-469, 2013.

[23] Scott Krig, "Computer Vision Metrics Survey, Taxonomy, and Analysis", ISBN: 978-1-4302-5929-9, Apress Berkeley, CA, 2014.

[24] E.R. Davies, "Computer Vision Principles, Algorithms Applications, Learning", Fifth Edition, Copyright (C) 2018 Elsevier Inc, ISBN: 978-0-12-809284-2, 2018

[25] Mikolajczyk, K., and C. Schmid. "A Performance Evaluation of Local Descriptors." Pattern Analysis and Machine Intelligence, IEEE Transactions on, Volume: 27, Issue: 10, 2005.

[26] Gauglitz, Steffen, Tobias Hollerer, and Matthew Turk. "Evaluation of Interest Point Detectors and Feature Descriptors for Visual Tracking." International Journal of Computer Vision Volume 94 Issue 3, September 2011.

[27] Andres Marmol, Thierry Peynot, Anders Eriksson, "Evaluation of Keypoint Detectors and Descriptors in Arthroscopic Images for FeatureBased Matching Applications", IEEE ROBOTICS AND AUTOMATION LETTERS, VOL. 2, NO. 4, OCTOBER 2017.

[28] G. Xinting, Z. Wenbo, F. Sattar, R. Venkateswarlu, and E. Sung, "Scale-space Based Corner Detection of Gray Level Images Using Plessey Operator," in Proc. of the Fifth International Conference on Information, Communications and Signal Processing, 2005, pp. 683-687. [29] G. Xinting, F. Sattar, and R. Venkateswarlu, "Multiscale Corner Detection of Gray Level Images Based on Log-Gabor Wavelet Transform," IEEE Transactions on Circuits and Systems for Video Technology, vol. 17 , pp. $868-875,2007$

[30] Moravec, H.P., "Towards automatic visual obstacle avoidance", 5 th International Joint Conference on Artificial Intelligence, pp. 584-594, 1977

[31] H. Moravec, "Obstacle Avoidance and Navigation in the Real World by a Seeing Robot Rover", Tech Report CMU-RI-TR-3 Carnegie-Mellon University, Robotics Institute, 1980.

[32] C. Harris and M. Stephens, "A combined corner and edge detector," in Alvey Vision Conference, pp. 147-151, 1988.

[33] Zhenxing Luo, "Survey of Corner Detection Techniques in Image Processing", International Journal of Recent Technology and Engineering (IJRTE), ISSN: 2277-3878, Volume-2, Issue-2, May 2013.

[34] Schmid, Cordelia, and Roger Mohr., "Local Gray Value Invariants for Image Retrieval", PAMI, V19, No 5, 1997).

[35] B. Sirisha and B. Sandhya, "Evaluation of distinctive color features from harris corner key points," in Proc. of the 2013 IEEE 3rd International Advance Computing Conference, pp.1287-1292, Feb, 2012.

[36] C. Schmid, R. Mohr, and C. Bauckhage, "Comparing and evaluating interest points," in Proceedings of the International Conference on Computer Vision, pp. 230-235, 1998.

[37] C. Schmid, R. Mohr, and C. Bauckhage, "Evaluation of interest point detectors," International Journal of Computer Vision, vol. 37, no. 2 , pp. 151-172,2000.

[38] Shi, J., and C. Tomasi., "Good Features to Track", Conference on Computer Vision and Pattern Recognition, 1994.

[39] Mikolajcyk, K., and C. Schmid, "An Affine Invariant Interest Point Detector", International Conference on Computer Vision, 2002.

[40] K. Mikolajczyk and C. Schmid, "Scale and affine invariant interest point detectors," International Journal of Computer Vision, vol. 1, no. 60, pp. 63-86, 2004.

[41] T. Tuytelaars and L. Van Gool, "Content-based image retrieval based on local affinely invariant regions," International Conference on Visual Information Systems, pp. 493-500, (C) Springer-Verlag Berlin Heidelberg, 1999

[42] T. Tuytelaars and L. Van Gool, "Matching widely separated views based on affine invariant regions," International Journal of Computer Vision, vol. 1, no. 59, pp. 61-85, 2004.

[43] Smith, S., Brady, J., "Susan-a new approach to low level image processing", Int. J. Comput. Vis., 23(1), 45-78, 1997. 
[44] Mahesh and M. V. Subramanyam, "invariant corner detection using steerable filters and harris algorithm" signal and Image Processing, An International Journal (SIPIJ) Vol.3, No.5, October, 2012.

[45] Li-hui Zou, Jie Chen, Juan Zhang, Li-hua Dou, "The Comparison of Two Typical Corner Detection Algorithms", Second International Symposium on Intelligent Information Technology Application, 2008

[46] Förstner, W; Gülch, "A Fast Operator for Detection and Precise Location of Distinct Points, Corners and Centres of Circular Features". ISPRS, 1987.

[47] T. Lindeberg, "Junction detection with automatic selection of detection scales and localization scales", Proc. 1st International Conference on Image Processing I. Austin, Texas, Pp: 924-928, 1994.

[48] Tony Lindeberg, "Feature detection with automatic scale selection", International Journal of Computer Vision, V 30, issue 2, pp: 77-116, 1998. [49] Banerjee M. and Kundu, M. K., "Handling of impreciseness in gray level corner detection using fuzzy set theoretic approach", Applied Soft Computing, 8(4), pp. 1680-1691, 2008.

50] Beaudet, P.R, "Rotationally invariant image operators", International Joint Conference on Pattern Recognition, pp. 579-583, 197.8

[51] Lakemond, R., Sridharan, S., Fookes, C., "Hessian-based affine adaptation of salient local image features", J. Math. Imaging Vis. 44(2), 150-167, 2012.

[52] Bay, Herbert, Andreas Ess, Tinne Tuytelaars, and Luc Van Gool, "Speeded-Up Robust Features (SURF)", Computer Vision and Image Understanding, Volume 110, Issue 3, Pages 346-359, June 2008.

[53] Mikolajczyk, K., Tuytelaars, T., Schmid, C., Zisserman, A., Matas, J., Schaffalitzky, F., Kadir, T., Gool, L., "A comparison of affine region detectors", Int. J. Comput. Vis., 65(1/2), 43-72, 2005.

[54] Mikolajczyk, K., and C. Schmid, "Indexing Based on Scale Invariant Interest Points", International Conference on Computer Vision, 2001.

[55] Rosten, E., Drummond, T., "Fusing points and lines for high performance tracking", International Conference on Computer Vision (ICCV'05), pp. 1508-1515. Beijing, China, 17-21 Oct, 2005.

[56] Rosten, E., Drummond, T., "FAST Machine learning for high speed corner detection", 9th European Conference on Computer Vision (ECCV’06), pp. 430-443. Graz, Austria, 7-13 May, 2006.

[57] Gunn, Steve R. "Edge Detection Error in the Discrete Laplacian of Gaussian." International Conference on Image Processing, ICIP. Proceedings, V 2, 1998.

[58] Kitchen, L., and A. Rosenfeld, "Gray-level Corner Detection", Pattern Recognition Letters Volume 1, issue: 2, pp: 95-102, 1982.

[59] Bretzner, L., and T. Lindeberg, "Feature Tracking with Automatic Selection of Spatial Scales", Computer Vision and Image Understanding Volume 71, Issue 3, Pages 385-392, September 1998.

[60] Lowe, David G., "Distinctive Image Features from Scale-Invariant Keypoints", International Journal of Computer Vision, Volume 60 Issue 2, Pages 91-110, 2004

[61] Kadir, T., and J. M. Brady, "Saliency, Scale and Image Description", International Journal of Computer Vision, Volume 45 Issue 2, Pages 83105, November 2001

[62] Kadir, Timor, Andrew Zisserman, and Michael Brady, "An Affine Invariant Salient Region Detector", European Conference on Computer Vision, 2004.

[63] Yussof, W., Hitam, M., "Invariant Gabor-based interest points detector under geometric transformation”, Digital Signal Process., V 25 pp: 190-197, 2014.

[64] Jackway, P.T., Deriche, M., "Scale-space properties of the multiscale morphological dilation-erosion", IEEE Trans. Pattern Anal. Mach. Intell. 18 (1), pp: 38-51, 1996

[65] Soille, P., "Morphological Image Analysis: Principles and Applications", 2nd edition, (C) Springer-Verlag Berlin Heidelberg, ISBN 978-3-642-07696-1, 2003 and 2004.

[66] Choudhary, R., Paliwal, J., Jayas, D.S., "Classification of cereal grains using wavelet, morphological, colour, and textural features of nontouching kernel images", Biosyst. Eng. 99, pp: 330-337, 2008.

[67] Le'zoray, O., Charrier, C., "Color image segmentation using morphological clustering and fusion with automatic scale selection", Pattern Recognit., Lett. 30 (4), pp: 397-406, 2009.

[68] Soille, P., Vogt, P., "Morphological segmentation of binary patterns", Pattern Recognit., Lett. 30 (4), pp: 456-459, 2009.

[69] Donoser, M., Bischof, H., "Efficient maximally stable extremal region (MSER) tracking", IEEE Comput. Soc. Conf., Comput. Vis. Pattern Recogn., Issue 1, pp: 553-560, 2006. 